\title{
A candidate liquid chromatography mass spectrometry reference method for the quantification of the cardiac marker 1-32 $B$-type natriuretic peptide
}

Article

Published Version

Creative Commons: Attribution-Noncommercial-No Derivative Works 3.0

Open Access Creative Commons Attribution-NonCommercial-NoDerivatives 3.0 License.

Torma, A. F., Groves, K., Biesenbruch, S., Mussell, C., Reid, A., Ellison, S., Cramer, R. and Quaglia, M. (2017) A candidate liquid chromatography mass spectrometry reference method for the quantification of the cardiac marker 1-32 B-type natriuretic peptide. Clinical Chemistry and Laboratory Medicine, 55 (9). pp. 1434-6621. ISSN 1437-4331 doi: https://doi.org/10.1515/cclm-2016-1054 Available at https://centaur.reading.ac.uk/70202/

It is advisable to refer to the publisher's version if you intend to cite from the work. See Guidance on citing.

To link to this article DOI: http://dx.doi.org/10.1515/cclm-2016-1054

Publisher: De Gruyter

All outputs in CentAUR are protected by Intellectual Property Rights law, including copyright law. Copyright and IPR is retained by the creators or other copyright holders. Terms and conditions for use of this material are defined in the End User Agreement. 


\section{www.reading.ac.uk/centaur}

\section{CentAUR}

Central Archive at the University of Reading

Reading's research outputs online 
Open Access

Attila F. Torma, Kate Groves, Sabine Biesenbruch, Chris Mussell, Alan Reid, Steve Ellison, Rainer Cramer and Milena Quaglia*

\section{A candidate liquid chromatography mass spectrometry reference method for the quantification of the cardiac marker 1-32 B-type natriuretic peptide}

DOI 10.1515/cclm-2016-1054

Received November 18, 2016; accepted March 9, 2017

\section{Abstract}

Background: B-type natriuretic peptide (BNP) is a 32 amino acid cardiac hormone routinely measured by immunoassays to diagnose heart failure. While it is reported that immunoassay results can vary up to $45 \%$, no attempt of standardization and/or harmonization through the development of certified reference materials (CRMs) or reference measurement procedures (RMPs) has yet been carried out.

Methods: B-type natriuretic peptide primary calibrator was quantified traceably to the International System of Units (SI) by both amino acid analysis and tryptic digestion. A method for the stabilization of BNP in plasma followed by protein precipitation, solid phase extraction (SPE) and liquid chromatography (LC) mass spectrometry (MS) was then developed and validated for the quantification of BNP at clinically relevant concentrations (15-150 $\mathrm{fmol} / \mathrm{g}$ ).

Results: The candidate reference method was applied to the quantification of BNP in a number of samples from the UK NEQAS Cardiac Markers Scheme to demonstrate its applicability to generate reference values and to preliminary evaluate the commutability of a potential CRM. The

\footnotetext{
*Corresponding author: Dr. Milena Quaglia, LGC Ltd., Science and Innovation, Queens Road, TW11 OLY Teddington, UK, E-mail: Milena.Quaglia@lgcgroup.com Attila F. Torma, Kate Groves, Sabine Biesenbruch and Steve Ellison: LGC Ltd., Teddington, UK Chris Mussell: LGC Ltd., Teddington, UK Alan Reid: UK NEQAS Cardiac Markers - Laboratory Medicine and FM Building, Queen Elizabeth University Hospital, Glasgow, UK Rainer Cramer: University of Reading - Department of Chemistry, Reading, Berkshire, UK
}

results from the reference method were consistently lower than the immunoassay results and discrepancy between the immunoassays was observed confirming previous data. Conclusions: The application of the liquid chromatography-mass spectrometry (LC-MS) method to the UK NEQAS samples and the correlation of the results with the immunoassay results shows the potential of the method to support external quality assessment schemes, to improve understanding of the bias of the assays and to establish RMPs for BNP measurements. Furthermore, the method has the potential to be multiplexed for monitoring circulating truncated forms of BNP.

Keywords: 1-32 B-type natriuretic peptide (BNP) reference method; brain natriuretic peptides; isotope dilution mass spectrometry; standardization.

\section{Introduction}

B-type natriuretic peptide (BNP) and N-terminal proBNP (NT-proBNP) are cardiac biomarkers secreted as proBNP, the prohormone of BNP, by the myocytes of the left ventricle in response to volume overload in an event of congestive heart failure [1]. In 2012, specific age-independent decision cut-offs for both BNP ( $\leq 100 \mathrm{ng} / \mathrm{L})$ and NT-proBNP $(\leq 300 \mathrm{ng} / \mathrm{L}$ ) were endorsed [2] and measurements of circulating BNP are currently used in clinical practice for the diagnosis of acute and chronic heart failure, risk stratification and monitoring response to therapy [3].

A number of immunoassays specific for BNP and NTproBNP analysis are commercially available and studies have been performed to assess their performance $[4,5]$. The results of the CardioOrmoCheck study, for example, highlighted discrepancy between immunoassays specific for BNP and discussed the requirement for measurement standardization [6]. 
A recognized route for standardization of clinical laboratory measurements is the development of reference measurement procedures including reference methods and certified reference materials (CRM) [7, 8]. In the biological area, the poor definition of the measurand has been identified as the major bottleneck and barrier for the development of reference methods and commutable reference materials. Immunoassays targeting BNP are currently calibrated with recombinant or synthetic BNP and the samples distributed by most of the quality assurance schemes are plasma spiked with BNP. It is, however, now accepted that proBNP, 1-32 BNP and NT-proBNP are not the only forms circulating [9-11]. The correlation between a reference method specific for what believed to be the measurand and immunoassays becomes in this case the first mandatory step to evaluate the potential of reference measurement procedures to improve measurement accuracy and precision in clinical laboratories.

Over the past decade a number of papers have been published on the development of methods for the quantification of BNP by mass spectrometry (MS) [10, 12-16]. Issues including the very low concentration of BNP in plasma, the difficulties associated with measuring large intact peptides by MS and poor stability of BNP in plasma (half-life of $22.6 \mathrm{~min}$ ) have however limited their further development for application in clinical laboratories.

Here we present for the first time the development of a reference liquid chromatography-mass spectrometry (LC-MS) method for quantification of BNP in plasma at clinically relevant concentrations. The method developed was applied to quantify BNP in a number of UK NEQAS Cardiac Markers Scheme samples and the correlation between the LC-MS results and immunoassay results was evaluated to define the utility of the method to establish reference measurement procedures for BNP.

\section{Materials and methods}

\section{Materials}

All materials were from Sigma-Aldrich (St. Louis, MO, USA) unless otherwise stated and details on the purity of the materials can be found in the Supplemental material file (A). Formic acid (FA) and Tris(2-carboxyethyl)phosphine hydrochloride $\left(\mathrm{TCEP}^{\star} \mathrm{HCl}\right)$ were from Thermo Fisher Scientific (Loughborough, UK), HCl from ROMIL (Cambridge, UK). N-methyl-N-(trimethylsilyl)-trifluoroacetamide (MSTFA) containing 1\% trimethylchlorosilane
(TMCS) from Restek Corporation (Belleforte, PA, USA). Modified sequencing grade trypsin was supplied by Roche Applied Science (Penzberg, Germany). L-methionine was from Fluka (Buchs, Switzerland). HPLC Optigrade organic solvents were from LGC (Teddington, UK).

The 32 amino acid BNP was from Sigma-Aldrich and the isotopically labeled BNP (SPKMV(13C5,15N)Q-G(13C2,15N)S-G(13C2,15N)-CFG(13C2,15N)-RKMDR-I(13C6,15N)-SSSSGLGC-K(13C6,15N2)-VLRRH) from CPC Scientific (Sunnydale, CA, USA). The average molecular weight was $3464.1020 \mathrm{~g} / \mathrm{mols}$. The lyophilized peptides were dissolved in water:acetonitrile $(90: 10 \mathrm{v} / \mathrm{v})$ containing $0.1 \% \mathrm{FA}(\mathrm{v} / \mathrm{v})$ and further dilutions were carried out in water containing $500 \mu \mathrm{g} / \mathrm{g}$ BSA and $0.5 \% \mathrm{FA}(\mathrm{v} / \mathrm{v})$. Certified amino acids from the National Metrology Institute of Japan (NMIJ, Tsukuba, Japan) were used for quantification of BNP.

MVQGSGCFGR, MVQGSGC-F(13C9,15N)-GR, ISSSSG-LGCK and ISSSS-G(13C2,15N)-L(13C6,15N)-GCK were from AnaSpec (Fremont, CA, USA) and in-house quantified against the NIST amino acid CRM (2389a) (NIST, Gettysburg, PA, USA). Human K2 ethylenediaminetetraacetic acid (EDTA) mixed gender plasma was purchased from Sera Laboratories International (Haywards Heath, UK).

\section{Methods}

Quantification of peptides: The method developed for the SI-traceable quantification of BNP and BNP tryptic digest peptide standards by amino acid analysis was a modification of a method previously described $[17,18]$. The samples were hydrolyzed at $190^{\circ} \mathrm{C}$ for $30 \mathrm{~min}$ in an ETHOZ EZ Microwave Digestion System (Milestone, Sorisole, Italy) equipped with a protein hydrolysis kit containing $30 \mathrm{~mL}$ of $6 \mathrm{M}$ hydrochloric acid $(\mathrm{HCl})$. Each hydrolysis included a reagent blank (solvents only), natural amino acids control, labeled amino acids control, a recovery vial, two calibration blends and two sample blends. The hydrolyzed samples were lyophilized, reconstituted in $80 \mu \mathrm{L}$ MSTFA and analyzed by gas chromatography-mass spectrometry (GC-MS) [18]. Three hydrolyses containing two sample blends, and two calibration blends were performed. Samples were independently prepared before each hydrolysis. The final results were the average from all amino acid results $(n=6)$.

Quantification of BNP using tryptic digestion was performed by isotope dilution mass spectrometry (IDMS) using isotopically labeled peptides as internal standards [17-19]. Tryptic digestion was optimized as described by Cryar et al. [18]. Ultimately $250 \mu \mathrm{L}$ of sample blends and calibration blends in $500 \mathrm{mM}$ TRIS buffer and $10 \mathrm{mM} \mathrm{Ca}^{2+}$ (pH 8.10) were reduced and alkylated with $200 \mathrm{nmol}$ TCEP $\left(37^{\circ} \mathrm{C}, 60 \mathrm{~min}\right)$ and $400 \mathrm{nmol}$ : S-methyl methanethiosulfonate (MMTS) (in the dark, $37^{\circ} \mathrm{C}, 30 \mathrm{~min}$ ). The samples were tryptic digested at $37^{\circ} \mathrm{C}$ for $2 \mathrm{~h}$ with two additions of $3.5 \mu \mathrm{g}$ of trypsin. A Q-TRAP 4000 (ABSciex, Framingham, MA, USA) coupled to a 1100 HPLC (Agilent Technologies Inc, Santa Clara, CA, USA) was used. Separation was achieved on a XBridge BEH C18 column $(3.5 \mu \mathrm{m}$, $2.1 \times 150 \mathrm{~mm}$, pore diameter of $130 \AA$ A ; Waters Corporation, Milford, $\mathrm{MA}, \mathrm{USA}$ ) at $60^{\circ} \mathrm{C}$. Flow rate was $100 \mu \mathrm{L} / \mathrm{min}$, injection volume 10 $\mu \mathrm{L}$. Mobile phases were: (A) water $0.1 \% \mathrm{FA} \mathrm{(v/v)}$ and (B) acetonitrile $0.1 \% \mathrm{FA}(\mathrm{v} / \mathrm{v})$. A linear gradient from 5 to $40 \%$ of (B) over $30 \mathrm{~min}$ was applied. Details of the MS settings are reported in the Supplemental material file (B). The following selective reaction monitoring 
(SRM) experiments were set up: 729.4/739.4 $\rightarrow 544.3 / 549.3$ for MVQGSGC(Methylthio)FGR/MVQGSGC(Methylthio)-F(13C9,15N)GR and 784.4/793.5 $\rightarrow$ 492.8/497.3 for ISSSSGLGC(Methylthio)K/ ISSSSG(13C2,15N)-L(13C6,15N)-GC (Methylthio)K.

LC-MS/MS analysis of BNP: The BNP stock solution was analyzed for potential impurities on an Agilent Q-TOF 6530 equipped with a JetStream (AJST) electrospray ionization (ESI) source. Quantification of BNP was performed on an Agilent 6490 Triple Quadrupole (QqQ) also equipped with an AJST ESI source. Both instruments were coupled to an Agilent 1290 UHPLC. Identical source and chromatographic conditions were used for the two sets of experiments and details of the source conditions are reported in the Supplemental material file (C). For the IDMS quantification of BNP by QqQ-SRM, experiments were set up by fragmenting the $6+$ charge state of the intact precursor ion (BNP: $m / z$ 578.3, BNP*: $m / z$ 583.3) and monitoring the $4+\mathrm{y} 26$ product ion (BNP: $m / z$ 699.2, BNP*: $\mathrm{m} / z$ 705.2) The dwell time was $100 \mathrm{~ms}$, fragmentor voltage 380.0 V. Collision energy (CE) and cell acceleration voltage were $18 \mathrm{~V}$ and $1 \mathrm{~V}$, respectively. All data were acquired in positive ion mode. Chromatographic separation was performed by using a Phenomenex Aeris PEPTIDE XB-C18 column $(2.6 \mu \mathrm{m} ; 2.1 \times 250 \mathrm{~mm}, 90 \AA$ $)$ at $60{ }^{\circ} \mathrm{C}$. A 20 -min linear gradient from 1 to $20 \%$ (B) was applied. Mobile phases were: (A) water $0.5 \% \mathrm{FA}(\mathrm{v} / \mathrm{v})$ and (B) acetonitrile $0.5 \% \mathrm{FA}(\mathrm{v} / \mathrm{v})$. The flow rate was $400 \mu \mathrm{L} / \mathrm{min}$. Dimethyl-sulfoxide (DMSO) was post-column infused at $1 \mu \mathrm{L} / \mathrm{min}$. Injection volume was $10 \mu \mathrm{L}$.

Sample clean-up: Details on sample clean up are provided in the Supplemental material file (D). Briefly, protein precipitation (PPT) was achieved by adding $1.5 \mathrm{~mL}$ of water:acetonitrile (20:80; v/v) containing $0.5 \% \mathrm{FA}$ to $0.5 \mathrm{~mL}$ of plasma samples. Solid phase extraction (SPE) was performed on a STRATA XL cartridge $(200 \mathrm{mg} / 3 \mathrm{~mL}, 300 \AA$, $100 \mu \mathrm{m}$; Phenomenex, Macclesfield, UK) by eluting BNP with $4 \mathrm{~mL}$ water:acetonitrile $(65: 35 ; \mathrm{v} / \mathrm{v})$ containing $0.5 \% \mathrm{FA}$.

\section{Results and discussion}

\section{Characterization and quantification of BNP primary calibrator}

The BNP stock solution was analyzed by LC-MS using a Q-TOF instrument to identify peptidic impurities which could compromise the accuracy of the quantification results. The main impurities identified by accurate mass $(\leq 4 \mathrm{ppm})$ were deletion products of BNP including a peptide missing one serine $(\mathrm{S})$ and one proline $(\mathrm{P})$ residue, peptides missing one $S$ residue and peptides missing one glycine $(G)$ residue. Peptides corresponding to BNP with oxidized methionine (positions 4 and 15) were also detected. An approximate calculation of the relative percentage of impurities was performed by assuming that the impurities had the same ionization efficiency as the BNP. The impurities missing both $\mathrm{S}$ and $\mathrm{P}$ were $~ 1.6 \%$ of the major peak, missing one $\mathrm{S}$ were $\sim 2.3 \%$ and one $\mathrm{G}$ were $1.5 \%$. The amount of oxidized BNP in the stock solution was less than $1 \%$.

The peptidic content of the BNP stock solution was SI traceably quantified by amino acid analysis as described in the Methods section. The results obtained are shown in Figure 1. It can be seen that the BNP concentration calculated from the amino acid $\mathrm{P}$ was lower than the BNP concentrations calculated from the other amino acids, but within uncertainty. This could be justified by the presence of the impurity missing the residues $\mathrm{S}$ and

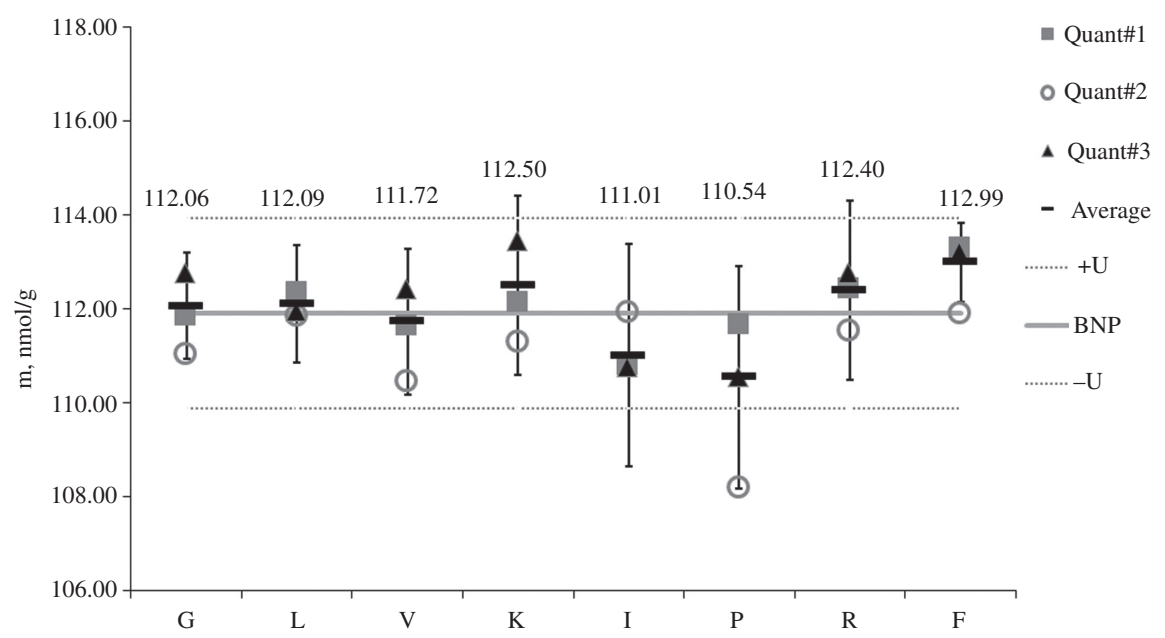

Figure 1: Results from the quantification of the BNP stock solution by amino acid analysis.

Each point represents the concentration of BNP in nmol/g [m(nmol/g)] calculated for each amino acid (G, L, V, K, I, P, R, F, x-axis) from three independent experiments (QUANT\#1, QUANT\#2 and QUANT\#3). The gray line (BNP) is the concentration of BNP calculated as the average of the amino acid results. The two dotted lines are the expanded uncertainties $(\mathrm{U}, \mathrm{k}=2)$ of BNP concentration calculated as described in the Supplemental material file (E). 
P (-SP) previously identified. No significant difference was observed for the value obtained from the amino acid glycine (G). All stable amino acids were included in the calculation of the concentration of the BNP stock solution (including proline and glycine), thus including the variability associated to the presence of peptidic impurities. The standard and combined uncertainties were calculated in accordance with the ISO Guide to the Expression of Uncertainty in Measurement [20] and EURACHEM guidelines [21]. Further purification of the BNP material, quantification of the impurities by using appropriate internal standards and a full purity assessment of the standard will improve accuracy and reduce uncertainty of the measurements and will have to be performed for the production of a CRM. The concentration of the BNP stock solution determined via amino acid analysis was confirmed by IDMS of the tryptic digested peptides using the isotopically labeled peptides as internal standards. A value of (111.92 \pm 2.03$) \mathrm{nmol} / \mathrm{g}$ was obtained by amino acid analysis and a value of $(100.63 \pm 10.69) \mathrm{nmol} / \mathrm{g}$ was calculated from the tryptic digestion approach. Both uncertainties are combined uncertainties (95\% confidence interval) and were calculated in accordance with the ISO Guide to the Expression of Uncertainty in Measurement [20] and EURACHEM guidelines [21]. The concentration of BNP from the amino acid analysis results was used for the quantification experiments of BNP in plasma. The overlap between the acid hydrolysis and tryptic digestion values confirms the accuracy of the amino acid analysis method here optimized. By combining the amino acid analysis experiments and the tryptic digestion experiments, $87 \%$ of the BNP sequence was quantified. The agreement within the calculated uncertainty of the quantification results from the individual amino acid and the tryptic digest peptides suggests that further purification would mostly reduce measurement uncertainty. While full purity assessment experiments of the standard are on-going, the uncertainty here obtained and the material were considered fit-forpurpose for this study.

\section{Quantification of BNP in plasma}

Analysis of large peptides/proteins by ESI MS is facilitated by the formation of multiply charged ions. This, however, renders the detection of large peptides at low concentration (e.g. pg/g and fmol/g) challenging as the ion abundance, and thus signal, is split between multiple charge states. An iterative optimization of sample clean up, LC and MS was performed to achieve the limit of detection required in plasma.
The use of "superchargers" [22-26] as additives to the mobile phase was investigated to increase signal intensities and ultimately enable quantification of BNP at clinically relevant concentrations. It has been reported that the addition of superchargers increases the charge state of macromolecules and enhances signal intensities through improving ionization efficiency [22-26]. Superchargers are additives (often to the LC's mobile phase) with a high boiling point which affects the surface tension of the droplets produced in electrospray. This leads to an increased charge density before droplet fission.

Three supercharging reagents were evaluated: DMSO, m-nitrobenzyl alcohol (mNBA) and sulfolane. To avoid contamination of the LC system and potential changes of the chromatographic elution profile, all superchargers were post-column infused at $1 \mu \mathrm{L} / \mathrm{min}$ by using a second isocratic pump. In Figure 2, the results are reported that were obtained by injecting BNP in the absence and presence of the three supercharging reagents. It can be observed that without any superchargers and in the presence of DMSO the most intense charge state is $6+$ and that the signal intensity for the $6+$ ion is the highest in the presence of DMSO. Dimethyl sulfoxide was therefore selected for further experiments and the increase of ion signal induced by infusing DMSO at $1 \mu \mathrm{L} / \mathrm{min}(0.25 \%)$ and at $5 \mu \mathrm{L} / \mathrm{min}(1.25 \%)$ was compared. As no further ion signal increase was observed by infusing DMSO at higher percentages, DMSO was infused at $1 \mu \mathrm{L} / \mathrm{min}(0.25 \%)$.

The analytical column was then substituted with a $2.6 \mu \mathrm{m}$ fused core C18 silica particle column. This, together with further optimization of the LC and MS conditions enabled the detection of BNP in aqueous solutions at $6 \mathrm{fmol} / \mathrm{g}(20.8 \mathrm{pg} / \mathrm{g})$.

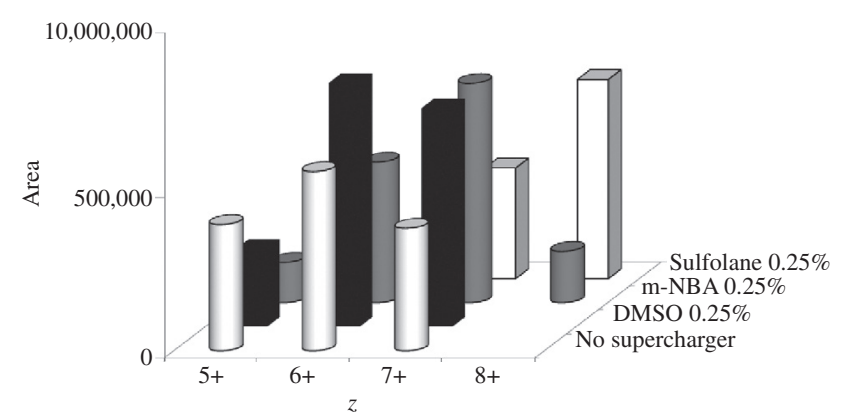

Figure 2: MS ion signal peak areas of the most intense charge states (z) detected when analysing $100 \mathrm{pmol} / \mathrm{g}$ of BNP in full-scan MS mode ( $m / z$ 400-800) by infusing superchargers.

The white cylindrical columns represent the integrated areas of the different charge states of BNP without any supercharger infused. The other columns represent the integrated areas of the different charge states of BNP with superchargers. 
A two-step sample clean-up procedure (PPT and SPE) was also optimized to enable detection and quantification of BNP in plasma.

SPE conditions were initially optimized by using aqueous BNP solutions. Although the calculation of the recovery of BNP is not a requirement for accurate quantification by IDMS, low recovery compromises method sensitivity. An approximate calculation of the recovery of BNP during sample clean-up was therefore performed to facilitate method development by post-spiking isotopically labelled BNP at the same concentration as BNP pre-spiked.

The STRATA XL cartridges showed the best retention properties for BNP and were selected for further optimization. After conditioning, a volume of $100 \mu \mathrm{L}$ of an aqueous BNP solution (1 pmol/g) was loaded and elution was performed with the sequential addition of $2 \mathrm{~mL}$ of water containing increasing percentages of acetonitrile (increments of $10 \%$ from 10 to $100 \%$ [v/v]). The washing and elution solutions were freeze-dried and reconstituted in water 0.5 $\%$ FA (v/v). BNP was detected in the $20 \%-40 \%$ acetonitrile elution steps with a recovery below $50 \%$. The poor recovery of BNP was found to be partially caused by oxidation of BNP occurring during SPE. Whereas the use of the labeled internal standard compensates for any bias during quantification, oxidation of BNP compromises the sensitivity of the method and therefore has to be reduced. The addition of $45 \mu \mathrm{g} / \mathrm{g}$ of methionine as a scavenger to the washing and elution solvents reduced BNP oxidation below $1 \%$.

A PPT method was optimized as the first sample clean-up step for quantification of BNP in plasma to remove most of the high-abundance proteins present and enable detection of BNP in plasma. In order to do this, the amount of acetonitrile in the precipitation solvent was varied to maximize recovery and minimize matrix suppression The optimal PPT solvent was water:acetonitrile 65:35 (v/v). The recovery calculated with the optimized conditions was 58\%. The addition of FA during PPT was performed to inhibit the activity of the enzymes involved in the degradation of BNP in plasma $[27,28]$.

\section{Stabilization of BNP}

In order to verify the stabilization effect of reducing the $\mathrm{pH}$ of plasma to block protease activity, 10 aliquots containing $60 \mathrm{fmol}(209 \mathrm{pg}$ ) of BNP were freeze-dried and $0.5 \mathrm{~g}$ plasma was added gravimetrically to each aliquot. The precipitation solvent was added at time $0(T 0)$ to the first aliquot and at intervals of $10 \mathrm{~min}$ to the remaining aliquots. Samples were subjected to SPE, reconstituted in water $0.5 \% \mathrm{FA}(\mathrm{v} / \mathrm{v})$ containing an equimolar amount of labeled BNP and analyzed by LC-MS/MS. The ratios of natural/isotopically labeled BNP are reported in Figure 3 and show the degradation of BNP over time in the absence of the PPT solvent. From this, it is evident that the amount of intact BNP decreases rapidly in plasma. The half-life of BNP in plasma was found to be $22.6 \mathrm{~min}$ and agrees with the half-life previously reported [29]. However, when the PPT solvent was added to the plasma, BNP did not degrade during clean-up and after 2 days stored at $5^{\circ} \mathrm{C}$ in the LC autosampler, showing the efficacy of the stabilization protocol utilized.

\section{Method validation}

The limit of detection and quantification, repeatability, intermediate precision, linearity and accuracy of the method were assessed. Three sets of eight plasma samples including a blank containing labelled BNP at $209 \mathrm{pg} / \mathrm{g}$ and an increasing amount of BNP primary calibrator (15$150 \mathrm{fmols} / \mathrm{g}$; 52-520 pg/g) were gravimetrically prepared. Samples were processed as previously described. The MS ion signal area ratio between the natural and labelled BNP $\left(A R E A_{\mathrm{NATBN}} / A R E A_{\mathrm{LAB} \mathrm{BNP}}\right)$ was corrected for the gravimetric amount of labelled BNP added. The corrected ratios were plotted against the gravimetric amount of BNP in each sample $\left(m_{\mathrm{NAT}}[\mathrm{pg}]\right)$ to obtain a calibration curve. The calibration points from the three experiments were combined to construct the calibration curve displayed in Figure 4 and the results are reported in Table 1.

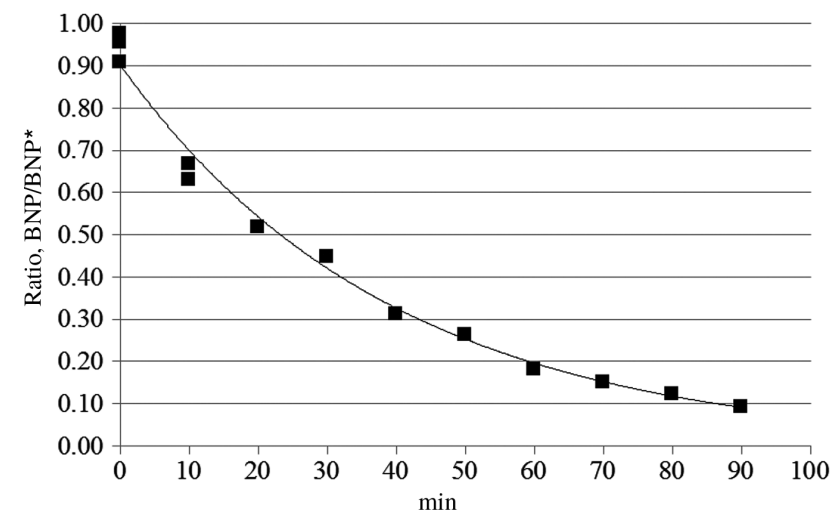

Figure 3: BNP degradation profile in the absence of the stabilizing PPT solvent.

The SRM MS ion signal ratios of natural (BNP) vs. isotopically labeled BNP (BNP*) are plotted against the time BNP spent in contact with plasma without the addition of the PPT solvent. 


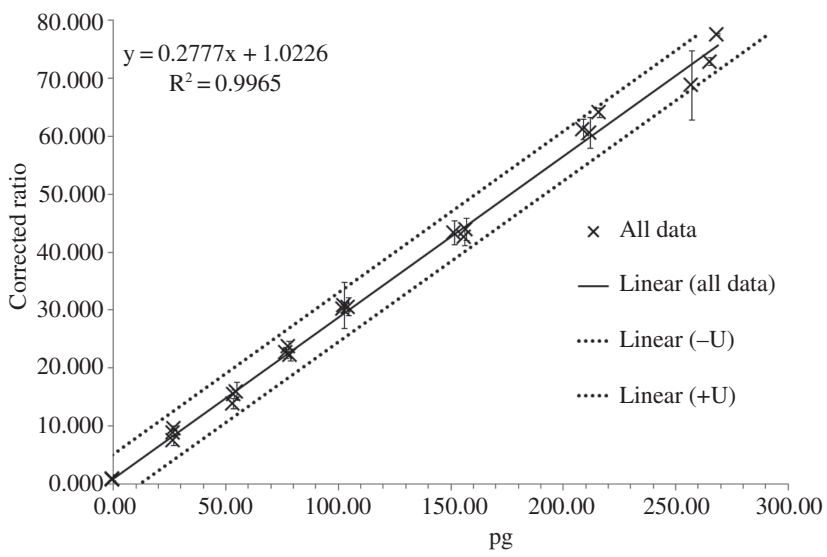

Figure 4: Calibration curve obtained from the data points of three independent sets of samples.

Each point represents the average of three injections.

The accuracy of the method was assessed by calculating the difference (bias) between the amounts of BNP determined from the constructed linear regression curve with the gravimetric SI-traceable concentration values. This was performed by considering each point of the calibration point as an unknown sample and by quantifying its BNP content using the calibration curve obtained with the remaining points. The repeatability was calculated as the average $(n=3)$ standard deviation of replicate injections $(n=3)$ and the intermediate precision was calculated as the standard deviation of the individual results at each level and reported as relative standard deviations in Table 1.

The calculation of the random error associated with both the intercept and the slope was performed to determine the uncertainty associated to the measurements performed by linear calibration $\left(x_{0}\right)$ [30]. The confidence limits were calculated by expanding the standard deviation of the determined concentrations $\left(S_{x o}\right)$ with the t-value at $95 \%$ confidence level, with (n-2) degrees of freedom. Where $n$ is the number of calibration points.

$$
x_{0} \pm t_{(n-2)} s_{x_{0}}
$$

Analysis of variance (ANOVA) of the calculated concentrations at each level from experiments performed on different days confirmed that the inter-day variability is not statistically significant.

The recovery of BNP from plasma was $58 \%$ as previously reported. However because an isotopically labelled internal standard was added before sample clean up, recovery was not calculated for each experiment as not affecting the quantification results.

A limit of detection of $4.37 \mathrm{fmols} / \mathrm{g}(15.15 \mathrm{pg} / \mathrm{g})$ was measured by calculating the standard deviation of the analytical background response of blank samples $(n=9)$. Fifteen femtomoles/gram ( $52 \mathrm{pg} / \mathrm{g}$ ) was considered the lowest limit of quantification based on visual evaluation as the minimum level at which the analyte can be quantified with acceptable accuracy and precision as shown in Table 1.

\section{Analysis of UK NEQAS samples}

The method above described was applied to samples from the UK NEQAS Cardiac Markers Scheme. Three samples were received monthly and processed after receipt. The lyophilized plasma samples containing unknown amount of BNP were reconstituted by following the instructions provided by UK NEQAS. One and half microLitre of the PPT solvent was added to each sample upon reconstitution. Seven plasma samples spiked with the BNP primary calibrator in a range of concentrations between $15 \mathrm{fmol} / \mathrm{g}$ and $155 \mathrm{fmol} / \mathrm{g}$ and a blank were also prepared and used to construct the calibration curves for each set of samples.

Table 1: Concentration levels for the calibration curve and calculated statistics from the validation experiments.

\begin{tabular}{lrrrr}
\hline pg/ga Plasma & $\mathbf{\pm U}$ & Bias $^{\text {b }}, \mathbf{p g} /$ g plasma & Repeatability & Intermediate precision \\
\hline 0.00 & 15.49 & - & - & - \\
53.99 & 15.41 & 6.86 & 4.57 & 7.92 \\
107.97 & 15.36 & 9.82 & 7.87 & 9.83 \\
155.85 & 15.33 & 7.21 & 7.07 & 7.85 \\
206.71 & 15.31 & 11.28 & 14.81 & 15.71 \\
310.21 & 15.34 & 10.51 & 13.34 & 12.29 \\
425.01 & 15.47 & 20.80 & 12.78 & 16.73 \\
527.56 & 15.62 & 33.28 & 16.49 & 34.60 \\
\hline
\end{tabular}

${ }^{\mathrm{a}}$ Gravimetrically prepared concentration of BNP in pg/g; ${ }^{\mathrm{b}}$ Expanded uncertainty calculated as described in the Supplementary material Section E. 
Thirty femtomole of isotopically labeled BNP were added to the calibrants and the UK NEQAS samples. All samples were processed and analysed as previously described by performing the two-step sample clean-up procedure prior to LC-MS/MS analysis. The results from the calibrants were plotted as the gravimetric amount of primary calibrator vs. the MS area ratios of the natural BNP divided by the isotopically labeled BNP. The amount of BNP in the UK NEQAS samples was calculated from the mass spectrometric ratio natural/isotopically labeled BNP.

The comparison of the LC-MS results with the results obtained from three different assays collated through the UK NEQAS scheme is shown in Figure 5. The results are in agreement with the literature where high cross-reactivity of assays with BNP metabolites and circulating proBNP lead to overestimation of the amount of BNP present in plasma samples. Furthermore, poor characterization of calibrants and different specificity of the assays are considered the major causes of inter-assay discrepancy [31]. As the LC-MS method is a targeted method for the 1-32 BNP and therefore does not suffer from interferences which may be due to the presence of metabolites or proBNP, lower results are to be expected. It has to be however highlighted that the UK NEQAS samples are stabilized plasma samples spiked with BNP. If fragments of BNP are present as residues in the depleted plasma before spiking or as a result of degradation of BNP post-spiking, their relative amount should be constant and discrepancies between the assays should be attributed to poor characterization of the immunoassay calibrants and different assay specificity. In order to confirm this, the same samples were sent more than once through the scheme (e.g. S1-S2; S4-S6; S7-S8; S10-S12; S11-S15) and the consistent results obtained by LC-MS and immunoassays show that the measurement bias between assays is not due to a random degradation of the spiked samples. The presence of minor percentages of degradation products in the samples was confirmed by developing an SRM method to monitor for the presence of the fragments 3-32, 3-30 and 4-32. The presence of those fragments in the UK NEQAS Cardiac Markers Scheme samples could be due to minor but consistent degradation of the spiked BNP into the samples or residues of the fragments in the plasma before spiking. As the BNP used for spiking plasma samples is a synthetic BNP, peptides with portions of the amino acid sequence of BNP could be both metabolites and/or impurities of the synthetic BNP. The SI-traceable quantified BNP primary calibrator with a known set of impurities was sent to UK NEQAS to be used for the preparation of plasma spikes and distributed through the Cardiac Markers Scheme. Whereas a slightly lower uncertainty was observed when the BNP primary calibrator was employed, the agreement between the different assays did not improve, suggesting that the discrepancy between assays is not caused by the presence of large amount of impurities in the BNP standard used to prepare the UK NEQAS Cardiac Markers Scheme samples.

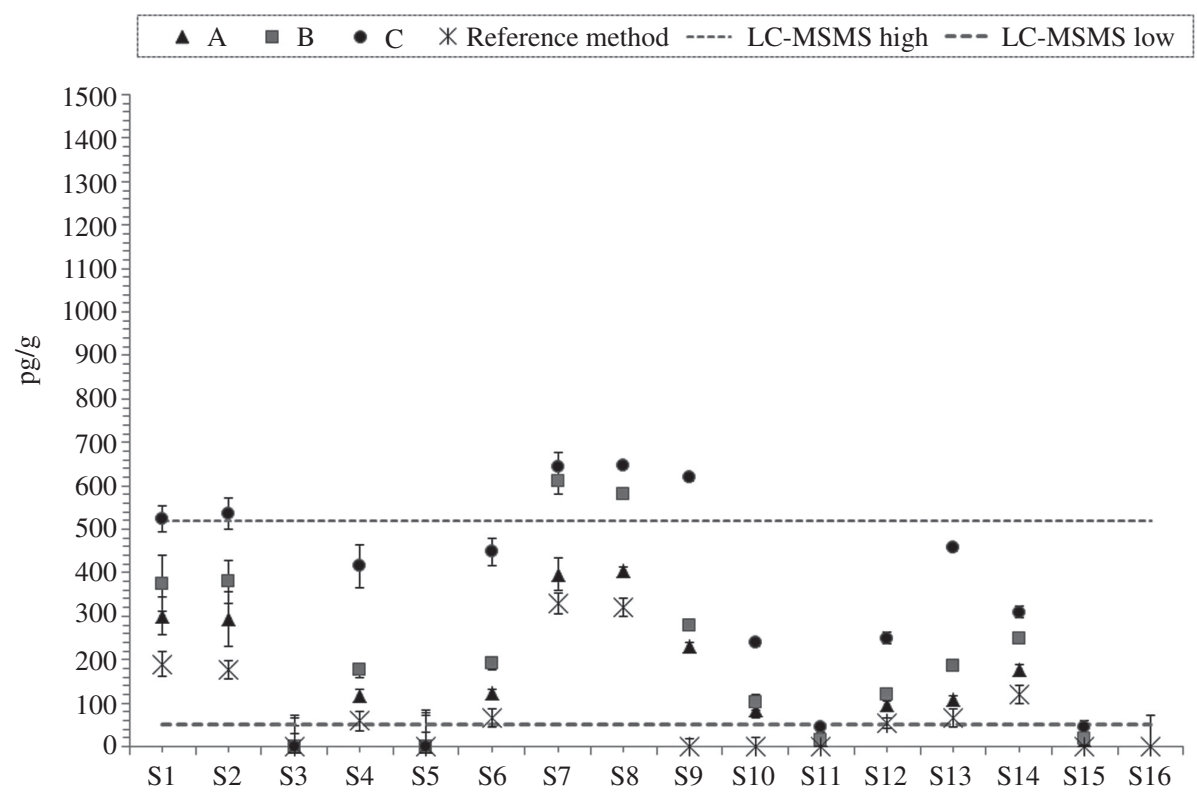

Figure 5: Immunoassay and LC-MS/MS results of the quantification of BNP in samples from the UK NEQAS Cardiac Marker Scheme. S1 and S2, S3 and S5, and S4 and S6 were the same samples sent blind twice through the scheme. The two dash lines (LC-MSMS HIGH and LC-MSMS LOW) represent the highest and lowest limits of the linearity range of the LC-MS method. 
A Passing-Bablok regression [32] was finally carried out to visualize the correlation between the LC-MS data and the immunoassay data (Figure 6). A correlation was observed between the LC-MS data and the data from the three assays with larger bias for assay $\mathrm{C}$.

\section{Conclusions}

Here we developed a method for SI traceable quantification of BNP in plasma at clinically relevant concentrations $(52-520 \mathrm{pg} / \mathrm{g})$ to be applied to underpin standardization of the cardiac marker BNP measurements through provision of reference values.

The quantification of the UK NEQAS Cardiac Markers Scheme samples by using the candidate reference method showed that the reference method results correlate with the immunoassay results particularly for the assays A and $\mathrm{B}$. The method has the potential to support external quality assessment schemes, to improve understanding of the bias of immunoassays and to establish reference measurement procedures for BNP. Further experiments are underway to collect and analyze patient samples with the ultimate goal to assess the suitability of the method to produce commutable CRM.

The availability of an LC-MS reference method for monitoring intact BNP that can be multiplexed to analyse BNP metabolites may offer a powerful tool to improve the understanding of this promising but disputed biomarker. It can be used to generate reliable data to support clinical trials of novel therapeutics targeting enzymes involved in the BNP metabolism [33, 34]. The development of standards and reference measurement procedures may finally
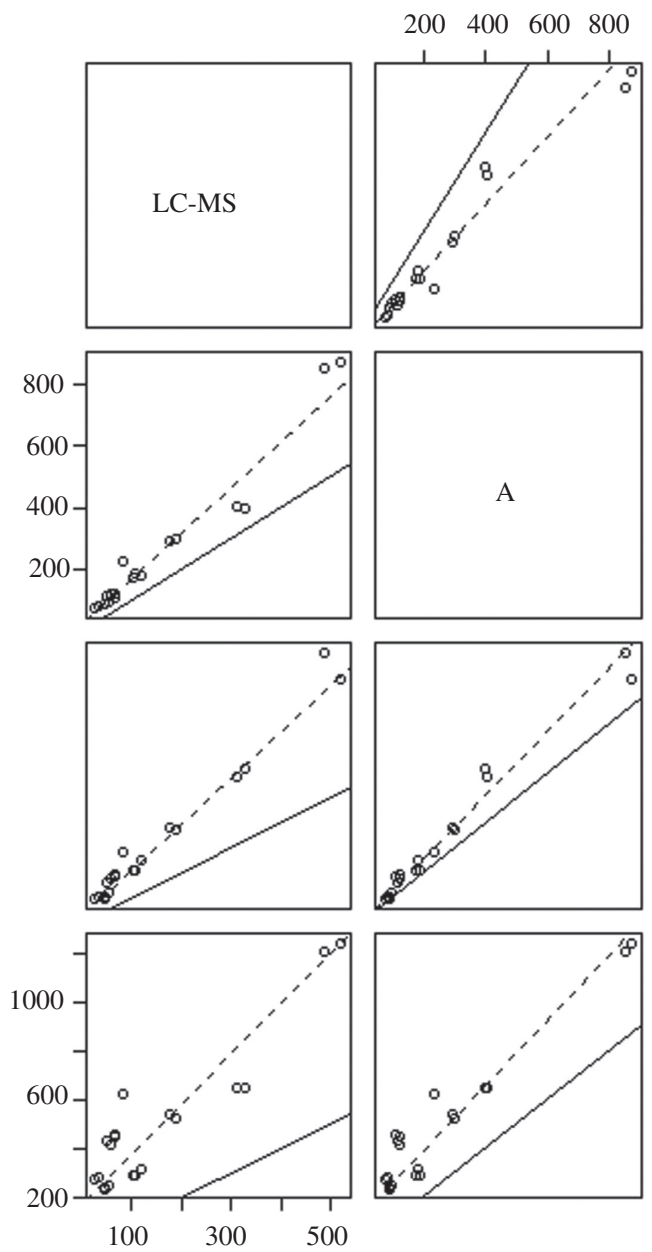
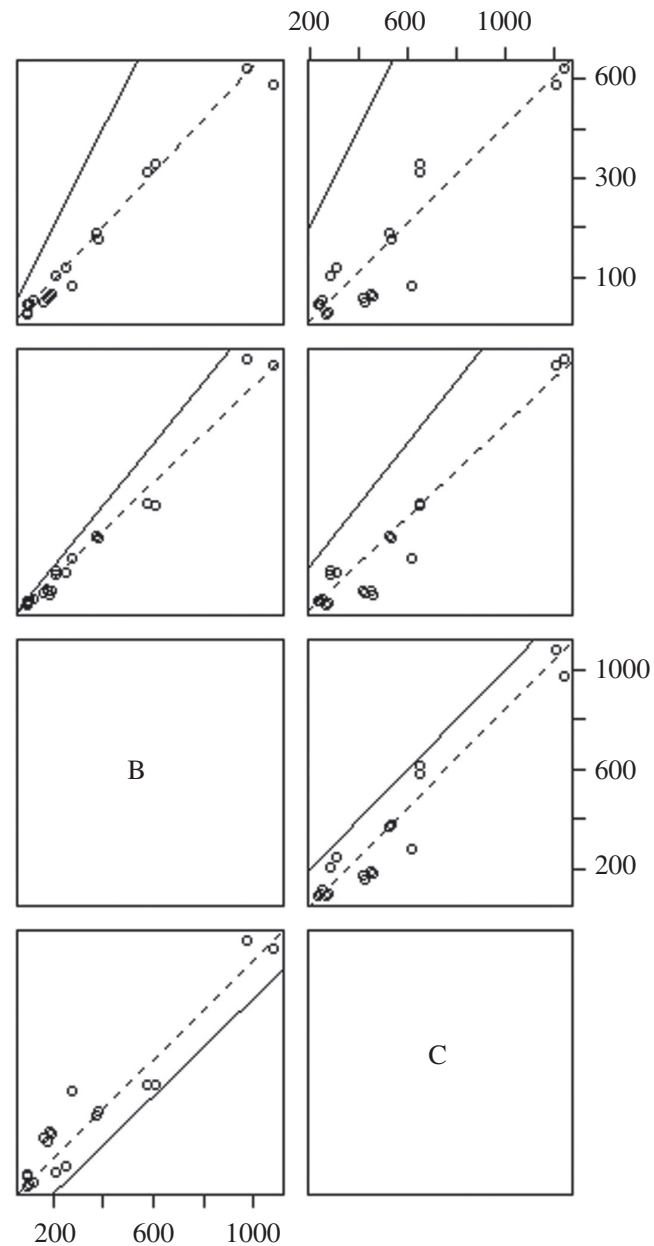

Figure 6: Results of the Passing-Bablok regression performed to visualise the correlation between the reference method (LC-MS) vs. immunoassays ( $A, B$ and $C$ ).

The dash lines represent the regression from the Passing-Bablock. The plain lines are the theoretical equivalence lines (score 1 and intercept 1). On the $x$ and $y$ axes are reported the values in $\mathrm{pg} / \mathrm{g}$ of BNP obtained by LC-MS and assay A, B and C. 
facilitate the regulatory approval of LC-MS based in vitro diagnostic devices (IVDs) for monitoring intact BNP and its metabolites [35].

Acknowledgments: The authors would like to thank Dr. Paul Collinson (St. George's Hospital, London) for discussing the standardization requirements in the area.

Author contributions: All the authors have accepted responsibility for the entire content of this submitted manuscript and approved submission.

Research funding: This work was supported by the UK Government Department for Business, Innovation and Skills.

Employment or leadership: None declared.

Honorarium: None declared.

Competing interests: The funding organization(s) played no role in the study design; in the collection, analysis, and interpretation of data; in the writing of the report; or in the decision to submit the report for publication.

\section{References}

1. Semenov AG, Tamm NN, Seferian KR, Postnikov AB, Karpova NS, Serebryanaya DV, et al. Processing of Pro-B-type natriuretic peptide: furin and corin as candidate convertases. Clin Chem 2010;56:1166-76.

2. Members AF, McMurray JJ, Adamopoulos S, Anker SD, Auricchio A, Böhm M, et al. ESC Guidelines for the diagnosis and treatment of acute and chronic heart failure 2012. Eur Heart J 2012;33:1787-847.

3. Roberts E, Ludman AJ, Dworzynski K, Al-Mohammad A, Cowie MR, McMurray JJ, et al. The diagnostic accuracy of the natriuretic peptides in heart failure: systematic review and diagnostic meta-analysis in the acute care setting. $\mathrm{Br}$ Med J 2015;350:h910.

4. Tamm NN, Seferian KR, Semenov AG, Mukharyamova KS, Koshkina EV, Krasnoselsky MI, et al. Novel immunoassay for quantification of brain natriuretic peptide and its precursor in human blood. Clin Chem 2008;54:1511-8.

5. Clerico A, Zaninotto M, Prontera C, Giovannini S, Ndreu $R$, Franzini $M$, et al. State of the art of BNP and NT-proBNP immunoassays: the CardioOrmoCheck study. Clin Chim Acta 2012;414:112-9.

6. Franzini M, Masotti S, Prontera C, Ripoli A, Passino C, Giovannini S, et al. Systematic differences between BNP immunoassays: comparison of methods using standard protocols and quality control materials. Clin Chim Acta 2013;424: 287-91.

7. Greg Miller W, Myers GL, Lou Gantzer M, Kahn SE, Schonbrunner ER, Thienpont LM, et al. Roadmap for harmonization of clinical laboratory measurement procedures. Clin Chem 2011;57: 1108-17.

8. Zegers I, Schimmel H. To harmonize and standardize: making measurement results comparable. Clin Chem 2014;60:911-3.
9. Seferian KR, Tamm NN, Semenov AG, Mukharyamova KS, Tolstaya AA, Koshkina EV, et al. The brain natriuretic peptide (BNP) precursor is the major immunoreactive form of BNP in patients with heart failure. Clin Chem 2007;53:866-873.

10. Niederkofler EE, Kiernan UA, O'Rear J, Menon S, Saghir S, Protter $A A$, et al. Detection of endogenous B-type natriuretic peptide at very low concentrations in patients with heart failure. Circ Heart Fail 2008;1:258-264.

11. Vasile VC, Jaffe AS. Natriuretic peptides and analytical barriers. Clin Chem 2017;63:50-8.

12. Hawkridge AM, Heublein DM, Bergen HR, Cataliotti A, Burnett $J C$, Muddiman DC. Quantitative mass spectral evidence for the absence of circulating brain natriuretic peptide (BNP32) in severe human heart failure. Proc Natl Acad Sci U S A 2005;102:17442-7.

13. Keshishian H, Addona T, Burgess M, Mani DR, Shi X, Kuhn E, et al. Quantification of cardiovascular biomarkers in patient plasma by targeted mass spectrometry and stable isotope dilution. Mol Cell Proteomics MCP 2009;8:2339-49.

14. Vatansever B, Lahrichi SL, Thiocone A, Salluce N, Mathieu $M$, Grouzmann E, et al. Comparison between a linear ion trap and a triple quadruple MS in the sensitive detection of large peptides at femtomole amounts on column. J Sep Sci 2010;33:2478-88.

15. Sobhi HR, Vatansever B, Wortmann A, Grouzmann E, Rochat B. Generic approach for the sensitive absolute quantification of large undigested peptides in plasma using a particular liquid chromatography-mass spectrometry setup. J Chromatogr A 2011;1218:8536-43.

16. Chappell DL, Lee AY, Bernstein HS, Lassman ME, Laterza OF. Development and validation of an IA-LC/MS method to quantitate active and total B-type natriuretic peptide in human plasma. Bioanalysis 2016;8:2341-9.

17. Pritchard C, Quaglia M, Burkitt WI, Mussell C, O’Connor G, Parkes $\mathrm{H}$. Fully traceable absolute protein quantification of somatropin: allowing independent comparison of somatropin standards. Clin Chem 2009;55:1984-90.

18. Cryar A, Pritchard C, Burkitt W, Walker M, O'Connor G, Burns DT, et al. Towards absolute quantification of allergenic proteins in food - lysozyme in wine as a model system for metrologically traceable mass spectrometric methods and certified reference materials. J AOAC Int 2013;96:1350-61.

19. Burkitt WI, Pritchard C, Arsene C, Henrion A, Bunk D, O'Connor G. Toward Système International d'Unité-traceable protein quantification: from amino acids to proteins. Anal Biochem 2008;376:242-51.

20. ISO. Guide to the expression of uncertainty in measurement. 1st ed. Geneva, Switzerland: International Organisation for Standardisation, 1995.

21. Ellison SLR, Williams A. Eurachem/CITAC guide: quantifying uncertainty in analytical measurement, 3rd ed. 2012. ISBN 978-0-948926-30-3. Available at: www.eurachem.org.http:// www.eurachem.org/index.php/publications/guides/quam. Accessed: 6 Nov 2013.

22. lavarone AT, Williams ER. Supercharging in electrospray ionization: effects on signal and charge. Int J Mass Spectrom 2002;219:63-72.

23. Sterling HJ, Williams ER. Origin of supercharging in electrospray ionization of noncovalent complexes from aqueous solution. J Am Soc Mass Spectrom 2009;20:1933-43. 
24. Sterling HJ, Prell JS, Cassou CA, Williams ER Protein conformation and supercharging with DMSO from aqueous solution. J Am Soc Mass Spectrom 2011;22:1178-86.

25. Miladinović SM, Fornelli L, Lu Y, Piech KM, Girault HH, Tsybin YO. In-spray supercharging of peptides and proteins in electrospray ionization mass spectrometry. Anal Chem 2012;84:4647-51.

26. Hahne H, Pachl F, Ruprecht B, Maier SK, Klaeger S, Helm D, et al. DMSO enhances electrospray response, boosting sensitivity of proteomic experiments. Nat Methods 2013;10:989-91.

27. Brandt I. Dipeptidyl-peptidase IV converts intact B-type natriuretic peptide into its des-SerPro form. Clin Chem 2006;52: 82-7.

28. Yandle TG, Richards AM. B-Type natriuretic peptide circulating forms: analytical and bioactivity issues. Clin Chim Acta 2015;448:195-205.

29. Hahne H, Pachl F, Ruprecht B, Maier SK, Klaeger S, Helm D, et al. Renal, endocrine, and hemodynamic effects of human brain natriuretic peptide in normal man. J Clin Endocrinol Metab 1993;76:91-6.

30. Miller JN. Basic statistical methods for Analytical Chemistry. Part 2. Calibration and regression methods. A review. Analyst 1991;116:3-14.
31. Miller WG. Time to pay attention to reagent and calibrator lots for proficiency testing. Clin Chem 2016;62:666-7.

32. Zidan M, Thomas RL, Slovis TL. What you need to know about statistics, part II: reliability of diagnostic and screening tests. Pediatr Radiol 2015;45:317-28.

33. Januzzi JL. B-Type natriuretic peptide testing in the era of neprilysin inhibition:are the winds of change blowing? Clin Chem 2016;62:663-5.

34. Mair J, Lindahl B, Giannitsis E, Huber K, Thygesen K, Plebani $M$, et al. Will sacubitril-valsartan diminish the clinical utility of B-type natriuretic peptide testing in acute cardiac care? Eur Heart J Acute Cardiovasc Care 2016;pii 2048872615626355. DOI: $10.1177 / 2048872615626355$.

35. Lathrop JT, Jeffery DA, Shea YR, Scholl PF, Chan MM. US food and drug administration perspectives on clinical mass spectrometry. Clin Chem 2016;62:41-7.

Supplemental Material: The online version of this article (DOI: 10.1515/cclm-2016-1054) offers supplementary material, available to authorized users. 